\title{
Construction and Study of Teaching Management Execu- tion System in Colleges and Universities
}

\author{
Yang $\mathbf{L i}^{1}$ \\ ${ }^{1}$ Baoding radio and television University, \\ Baoding, China
}

\author{
Hai-yu Zhang ${ }^{2}$ \\ ${ }^{2}$ Baoding women's vocation school, \\ Baoding,China
}

\begin{abstract}
The construction of a perfect teaching management execution system has gradually become an effective means of training high quality talents and enhancing the core competitiveness. First, the connotation of teaching management execution system in colleges and universities is analyzed in-depth to understand its composing elements; then, the current situation faced by teaching management execution system is analyzed to determine the necessity of building a execution system; finally, the teaching management execution system in colleges and universities is constructed, hoping to promote the smooth implementation of teaching management and improve the level of teaching quality.
\end{abstract}

Keywords: Teaching management in colleges and universities; execution system; system construction

\section{Introduction}

The central work of the management in colleges and universities is teaching management, which has direct and important influence on teaching quality and teaching position. The ultimate goal of teaching management is to transform the teaching thought to personnel training, obtaining good results, which is inseparable from the correct execution. Modern management theory holds that the success of an organization is inseparable from the right strategy and correct execution, of which $70 \%$ depends on the correct execution. Thus, execution is the core competitiveness of an organization, and has an important role in the relevant activities of the organization. The construction of a perfect teaching management system has gradually become an effective means of training high quality talents and enhancing the core competitiveness. At present, many scholars have carried out a lot of researches on the teaching management execution system in colleges and universities. Literature [1-3] analyzed the basic conditions or factors of the teaching management execution system in colleges and universities, and then put forward concrete measures to improve the teaching management execution. Literature [4] analyzed ecological connotation and ecological dilemma of teaching management execution from the perspective of ecology theory, and then pointed out how to reconstruct the teaching management execution in the perspective of ecology. Literature [5] proposed reasons for the lack of teaching man- agement execution in colleges and universities, and then strengthened the teaching management execution from four aspects of administrators' concept and quality, teaching management system, model and effectiveness of decision execution. Literature [6] put forward corresponding execution measures for the educational administration department in colleges and universities through the four influencing factors, including the main body, object, goal and means. Literature [7] deeply analyzed the four elements of the teaching management execution system: the implementation objectives, mechanisms, processes and performance evaluation, and then proposed corresponding strategies. Literature $[8,9]$ built teaching management execution system respectively based on analyzing the basic constructing idea. Related researches have laid a solid theoretical foundation for this study, which have important reference value.

At first, this paper analyzes the connotation of teaching management execution system to cognize its constituent elements; secondly, the present situation of teaching management execution system in colleges and universities will be analyzed to determine the necessity of building a clear system; finally, on these basis, the teaching management execution system will be constructed to promote the smooth implementation of teaching management in colleges and universities, improving the level of teaching quality..

\section{The connotation of teaching management execution system}

\subsection{Execution and teaching management execution}

Executing refers to a process of taking effective action and achieving the mission requirements under the guidance of specific target. Execution is derived from the connotation of executing, representing a kind of ability, that is to say, execution is an ability to ultimately realize organizational goals by understanding policy decisions and directives from superior leaders, implementing relevant objectives and decisions, as well as taking active measures to deal with the changing environment in the process of execution. And teaching management execution refers to that in the process of teaching management, based on the teaching goals, the main bodies of teaching management perform the teaching plan firmly and effectively and complete tasks of teaching according to the teaching objective laws and school regulations. 


\subsection{The composing elements of teaching management execution system}

It is the essential prerequisite for the construction of teaching management execution system to make clear its composing elements. According to the connotation of teaching management execution and relevant materials, we can know that the teaching management execution system mainly contains four parts as follows: (1) The teaching management execution goal, which is basic of teaching management execution system, is the soul of all teaching activities, guiding the whole process of teaching management. If teaching management execution goal is vague or confused, and then we couldn't refine teaching management plans and tasks, unable to carry out teaching management effectively, finally, the teaching management execution is impossible. (2) The teaching management execution mechanism, which is the necessary guarantee for the execution goal, includes three aspects: personnel mechanism, requiring to choose the right person or team to complete the executive goal and clear their rights and obligations; management mechanism, requiring standardized and rationalized teaching management system with openness, fairness and impartiality; supervision mechanism, requiring to track, check, compare and analyze the teaching management execution goal and practice. (3) The teaching management execution process, which is the core of teaching management execution system, includes the execution process of teaching administrators and the execution process of teaching teams. The former is the ultimate embodiment of the execution of teaching administrators, requiring them to complete their duty and responsibility seriously, rigorously, creatively, timely and high-quality in the executive process; the latter refers to the execution of teaching management unit, inseparable from the joint efforts from each teaching administrator. (4) The teaching management execution assessment, which is an important measure to improve teaching management execution, and also the feedback link of teaching management execution system. It needs to assess all aspects of teaching management scientifically to understand the actual situation of the execution and to set the corresponding improvement measures.

\section{The current situation of teaching management ex- ecution system in colleges and universities}

\subsection{The existing problems of teaching management execution system}

(1) The human management consciousness of teaching management in colleges and universities is weak.

The management objects of teaching management activities in colleges and universities are teachers and students with subjective initiative and creativity, which are usually ignored by teaching administrators. Nowadays, the admin- istration-oriented management consciousness still occupies the dominant position, and teaching management is also in the state of "following the administrators", which means that the demand of teachers and students is not get effectively meet, seriously hindering the implementation of teaching management execution to a certain extent.

(2) The scientific and continuous teaching management system is absent.

At present, the relevant rules, regulations and management plans of teaching management usually lacks sufficient scientific proof before implementation, being not conducive to the implementation; while others are difficult to implement or the implementing process changes too frequently and lacks of continuity and good execution, eventually leading to system failure. For example, the inefficiency or even missing of motivating mechanism will greatly dampens teachers' initiative; the unreasonable teaching management organization structure will cause the asymmetry or even chaos among responsibility, right and profit, and eventually greatly hinder effective execution.

(3) The construction of teaching management execution culture is missing.

In most colleges and universities, the teaching management execution system has not yet formed, let alone the construction of teaching management execution culture. The lack of culture leads to the loose of the overall plan, and even a poor internal cohesive. Moreover, execution culture also fails to get everyone's approval, seriously affecting the efficiency of execution.

(4) The supervision and guidance contents of teaching management are single with hysteretic feedback.

Recently, both teachers and teaching administrators are less able to correct understand the important role of teaching management supervision and guidance. Moreover, the contents of guidance and supervision are relatively narrow, and the ways of guidance and supervision are limited to listening and checking, unable to carry out deep and comprehensive supervision and guidance. In addition, the information feedback process of supervision and guidance is complex and hysteretic, unable to play their true utility.

\subsection{The necessity of building teaching management execution system}

The teaching management execution is an important factor to enhance the competitiveness of colleges and universities, and also is the key to implement the concept of education, the purpose of running a school as well as the development strategy. Teaching administrators in colleges and universities are not only responsible for formulating teaching plans, providing teaching conditions and managing teaching activities, but also responsible for the implementation of teaching plan, the command and coordination of faculty teaching activities, as well as the evaluation of teaching quality. It is the high time to improve teaching management execution, with important practical significance. However, it is a systematic work to improve the teaching management execu- 
tion, requiring more coordination from many aspects. Therefore, it is necessary to construct the teaching management execution system in colleges and universities in order to carry out teaching management activities systematically and effectively and improve the execution level as well as the teaching quality.

\section{The construction of teaching management execution system in colleges and universities}

Through analyzing the connotation of teaching management execution system, we understand the current situation and clear the necessity of construction. Therefore, the following are measures to construct the teaching management execution system in colleges and universities.

\subsection{Establishing advanced teaching management con- cept}

The "people-oriented" teaching management idea should be established, laying the ideological foundation for the construction of execution system. This kind of ideology should firstly be approved by the general staff, and then realize the binds among schools, departments and individuals, and also increase the cohesion of teaching management activities, finally form the campus cultural atmosphere of execution. On the one hand, the management mode should divide the labor clearly, define the responsibilities and require collaboration, guaranteeing the effective and successful completion of each post work. On the other hand, according to the objectives, the management method could arrange schedule reasonably, realize the inspection and control of the key, and constantly revise the gap between the actual and planning.

\subsection{Creating a high-level teaching management organ- ization}

The smooth implementation of teaching management activities can't be separated from the excellent, united and highexecutive teaching management organization, and usually teaching administrators are the most important members of teaching management organization. Firstly, teaching administrators should have a higher ideological and political tutelage and continue to learn to enrich themselves; secondly, teaching administrators should have a high sense of responsibility, so that they can complete the execution goals of teaching management activities well and efficiently; thirdly, teaching management organization need to have a strong cohesion, which is inseparable from the sense of unity and cooperation of each teaching administrator; finally, teaching administrators should have the consciousness and ability of innovation, which is the progressing source of teaching management activities continually.

\subsection{Improving the teaching management execution evaluation system}

Teaching management execution evaluation system is conducive to ensuring the sustainable development of teaching management execution, through which we can timely find ineffective implementation and take measures to improve, ensuring the effective implementation of the execution system. In terms of evaluation standards and indexes, it is necessary to be designed individually according to different activities, being comprehensive, scientific and standard; In terms of the implementation of evaluation, the target management and daily management should be combined together to carry out information collection, analysis, feedback and control, forming a closed-loop system evaluation system finally.

\subsection{Establishing the safeguard mechanism of teaching management execution system}

The safeguard mechanism of teaching management execution system includes three aspects: one is the incentive and restraint mechanism, which not only provides material and spiritual rewards for relevant personnel of teaching management, but also builds appropriate constraints by ways of target responsibility and competition; second is the democratic mechanism, which can fully mobilize the enthusiasm of teachers to be involved in the work of teaching management, teaching reform and others to promote the democratization of teaching management; third is supervision and guidance mechanism, namely a two-way communicating information feedback mechanism by using a variety of ways such as regular or irregular, statistical sampling, indepth visits and so on to carry out supervision and guidance.

\subsection{Strengthening the technical support of teaching management execution system}

The technical support of teaching management execution system is aimed at improving the teaching management execution level by use of powerful informationalized teaching management platform. On the one hand, the informationalized platform can realize the informatization of teaching management, such as the informationalized operations of teaching plan management, performance management, student course management and so on, which can realize the personalized service, and optimize the process of teaching management. On the other hand, the informationalized platform can be used to communicate information, disclosure information and provide services etc., being conducive to the transmission and feedback of information.

\section{Conclusions}

The construction of teaching management execution system is a systematic work, and can't be separated from the cooperation between teaching administrators and teachers. It needs to proceed from many aspects to construct teaching 
management execution system, but here only focuses on five aspects of management idea, teachers' level, evaluation system, safeguard mechanism and technical support, hoping to promote the improvement of execution level and teaching quality in colleges and universities.

\section{References}

[1] Liu Shijie. Study on the Improvement of Higher Education Teaching Management Execution[J]. Guide to Business, 2014(22):140-141.

[2] Xiang Jinzhi. The Absence and Construction of Teaching Management Execution in Colleges and Universities[J]. Education Exploration, 2008(1):72-73.

[3] Li Chenqing. The Absence and Construction of Teaching Management Execution in Colleges and Universities[J]. Journal of Suzhou Education Institute, 2014, 17(5):60-61.

[4] Lu Wuxia. Reconstruction of Teaching Management Execution in Vocational Schools that Based on Ecological Visual[J]. Audit Education, 2011(10): 36-38.
[5] Chen Xia. The Absence and Strengthening of Teaching Management Execution in Colleges and Universities[J]. Heihe Education, 2012(7): 83-83.

[6] Wei Jianjian, Wu Tiejun, Mu Xiaoyong. Study on Execution Construction of Educational Administration Department in Colleges and Universities[J]. Journal of Suzhou University (Engineering Science Edition), 2008, 27(5):69-71.

[7] Zhou Chuansheng. Research on the Construction of Teaching Execution System in Colleges and Universities[J]. Journal of Shenyang Normal University (Social Science Edition), 2011, 35(6):126-129.

[8] Qu Bo, Liu Tuo. Design and Realization of University Teaching Administration Executive Ability System[J]. Research in Higher Education of Engineering, 2011,06: 116-120.

[9] Qu Bo, Hou Yongfeng, Liu Tuo, Wei Wangqiang, Zhang Ying. The Establishment of Teaching Management Execution System Based on the "Plan-controlassessment"[J]. China University Teaching, 2009,05: 67-69. 